\title{
Investigation into the local nature of rate of change of frequency in electrical power systems
}

\author{
Damien Doheny \\ Technological University Dublin, damien.doheny@tudublin.ie \\ Michael Conlon \\ Technological University Dublin, michael.conlon@tudublin.ie
}

Follow this and additional works at: https://arrow.tudublin.ie/engscheleart

Part of the Electrical and Computer Engineering Commons

\section{Recommended Citation}

D. Doheny and M. Conlon, "Investigation into the local nature of rate of change of frequency in electrical power systems," 2017 52nd International Universities Power Engineering Conference (UPEC), 2017, pp. 1-6, doi: 10.1109/UPEC.2017.8231982.

This Conference Paper is brought to you for free and open access by the School of Electrical and Electronic Engineering at ARROW@TU Dublin. It has been accepted for inclusion in Conference papers by an authorized administrator of ARROW@TU Dublin. For more information, please contact arrow.admin@tudublin.ie, aisling.coyne@tudublin.ie,gerard.connolly@tudublin.ie.

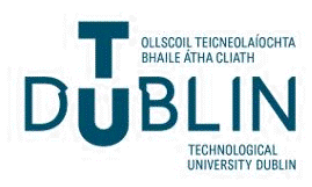




\section{Investigation into the Local Nature of Rate of Change of Frequency in Electrical Power Systems}

\author{
Damien Doheny \\ School of Electrical and \\ Electronic Engineering \\ Dublin Institute of Technology, Ireland \\ Email: damien.doheny@mydit.ie
}

\author{
Prof. Michael Conlon \\ School of Electrical and \\ Electronic Engineering \\ Dublin Institute of Technology, Ireland \\ Email: michael.conlon@dit.ie
}

\begin{abstract}
In recent years power systems world-wide have seen large increases in wind power penetration and this trend is expected to continue. This is having the undesirable consequence of reducing the inertia of electrical power systems, especially at times of high wind generation. Reduced inertia makes a power system more susceptible to a larger rate of change of frequency (RoCoF) following a grid disturbance, such as the sudden disconnection of a load or generator. High RoCoF events could trigger generator protective devices or anti-islanding RoCoF relays, disconnecting generation from the grid, compounding the initial grid disturbance and in extreme cases result in the cascade tripping of generators and grid blackout.

The objective of this research was to investigate how RoCoF varies with location in an electrical power system and determine if there is any significant difference between local RoCoF observed at individual buses and the system RoCoF seen across the entire power system. The results show that generators closest to the disturbance are impacted the most after the loss of a generator, and if this generator has relatively low inertia it could see a local RoCoF many times greater than the system RoCoF. It was also observed that when a large portion of the total power system inertia is concentrated at one machine, the mean of the local RoCoFs is significantly larger compared to when the power system inertia is equally distributed across all machines. It was observed that by measuring RoCoF using a rolling average window of 0.5 seconds, the magnitude of the measured $\mathrm{RoCoF}$ is significantly reduced and the effect that the distribution of inertia has on the mean of the local RoCoFs is eliminated. However, in some scenarios the local RoCof was still many times greater than the system RoCoF. The results demonstrate that local RoCoF could be an issue that needs to be considered when operating low inertia power systems, particularly as wind power continues to displace conventional generation.

Index Terms-Centre of Inertia, Inertia, Local Frequency,
\end{abstract} Local ROCOF, System Frequency, System ROCOF.

\section{INTRODUCTION}

Synchronous generators are traditionally used to generate and supply electrical energy to electrical power systems. However, as nations try to reduce $\mathrm{CO}_{2}$ emissions produced from conventional energy sources, greater levels of wind generation capacity is being connected to electrical power systems, displacing synchronous generators. Increased wind power penetration reduces total power system inertia and this makes frequency regulation more difficult [1], especially in the event of a disturbance on the electrical network, such as the sudden loss of a generator or load.
Of particular concern to transmission system operators (TSOs) is the rate of change of frequency (RoCoF) following a severe disturbance, such as the loss of the largest infeed generator. In a scenario where the wind penetration is high, system inertia is low and the largest in-feed generator is lost, the resulting RoCoF could be extremely high. This could trigger generator protective devices or anti-islanding RoCoF relays [2], disconnecting generation from the grid, compounding the initial grid disturbance and in extreme cases result in the cascade tripping of generators [3]. This risk is one of the factors that is limiting the maximum instantaneous wind penetration on the island of Ireland [4] and can cause wind curtailment [5]. Therefore, to ensure the safe and reliable operation of electrical power systems, while also maximising the instantaneous wind penetration, it is important that their behaviour following a disturbance is fully understood.

Some research has already been conducted on this subject. Creighton et al [6] looked at how increased levels of wind generation on the island of Ireland would impact the RoCoF in the event of grid disturbances. This research used computer models of the all Ireland transmission system to simulate typical three-phase fault scenarios. The RoCoF at four geographically displaced buses was observed following the simulated disturbances. The research showed that as the levels of wind generation increase, the magnitude of the RoCoF following a disturbance also increases. The results of the research also showed that the magnitude of the RoCoF can be significantly higher closer to the disturbance.

Similar research in this area has also been conducted by $\mathrm{Wu}$ et al [7]. This research used a computer model of the IEEE 39 bus system to investigate how the distribution of inertia affects the magnitude of RoCoF. It simulated the computer model under 10 different scenarios. In each scenario the total power system inertia was equal. However, the distribution of inertia was different in each scenario. The research concluded that the magnitude of RoCoF following a disturbance is also dependant on the distribution of inertia.

A common method used to predict the initial system RoCoF after a disturbance is based on the centre of inertia (COI) of the system as defined in [8]. A variation of this is presented in [9] as: 


$$
\frac{d f}{d t}=-\Delta P \frac{f}{2 H S}
$$

where $\frac{d f}{d t}$ is the initial RoCoF, $-\Delta P$ is the power imbalance because of the disturbance, $f$ is the system rated frequency, $H$ is the inertia constant of the entire system after the disturbance and $S$ is the rated power of the system. This method assumes that the power system can be considered as a single machine, possessing the total power system inertia and connected to a single bus with a single system frequency and RoCoF. However the results in [6] and [7] suggest that these assumptions are possibly too simplistic as this method does not take account of the local RoCoF at the individual generators or the distribution of the inertia.

TSOs must manage networks in such a way as to ensure that following a disturbance, the resultant RoCoF stays within acceptable limits. Currently in Ireland, generating units must remain synchronised to the grid following a disturbance, for a RoCoF up to $0.5 \mathrm{~Hz} / \mathrm{s}$ [10]. However, in anticipation of higher levels of wind generation, lower inertia on the system and potentially higher RoCoFs, EirGrid, the TSO in Ireland, has proposed to change the RoCoF standard to $1 \mathrm{~Hz} / \mathrm{s}$, measured over a rolling average window of $500 \mathrm{~ms}$ [3].

One possible strategy TSOs could use to prevent excessive RoCoFs and maintain them within limits, is to ensure there is always a minimum level of inertia connected to the system to counteract the worst possible disturbance, i.e. the loss of the largest in-feed generator. The minimum inertia could be determined using the COI method 1. However, this is possibly over-simplifying the problem as it involves a number of assumptions. Should these assumptions prove incorrect, it is possible that the RoCoF in a certain location of the network may be higher than expected, causing the disconnection of local generation, or it is also possible that the minimum levels of inertia are over-estimated, resulting in excessive wind curtailment.

The objective of this research was to investigate how RoCoF varies with location in an electrical power system following a disturbance and determine if there is any significant difference between local RoCoF observed at individual buses and the system RoCoF seen across the entire power system.

\section{ImPACt OF A Disturbance ON A POWER Systems}

The frequency of an electrical power system is dependent on the rotational speed of the synchronous generators; hence, their speed must be kept constant to maintain rated frequency. A steady state speed and frequency is achieved when the mechanical power input to the electrical generators equals the electrical power demand on the system. To maintain this power balance, elaborate control systems are used to regulate the mechanical input power. Should a sudden disturbance occur on the electrical power system, the control systems will not react quickly enough and a momentary power imbalance will exist, causing the generators speed and the power system frequency to deviate.
The magnitude of the power imbalance resulting from a disturbance determines how quickly the generators speed and system frequency changes. This is termed the Rate of Change of Frequency (RoCoF), measured in Hertz per second [Hz/s], and is used as a measure of the severity of a disturbance. The inertia of the rotating mass of an electrical generator plays an important role during a disturbance; it acts as a short-term energy storage medium and releases or absorbs energy while a power imbalance exists, reducing the RoCoF. The inertia of the individual generators connected to a power system contribute to the total power system inertia. In the context of electrical power systems, inertia is given the symbol $H$ and is measured in terms of its stored rotational energy in $[M W . s]$ or is sometimes referred to as the inertia constant and expressed in per unit form, with units of seconds $[s]$.

The fictitious inertial centre of a power system, as defined in [8], has an angle $\bar{\delta}$, an angular velocity $\bar{\omega}$ and mean acceleration $\frac{d \bar{\omega}_{\Delta}}{d t}$, given by;

$$
\begin{array}{r}
\bar{\delta}=\frac{\sum_{i=1}^{N_{g}} \delta_{i} H_{i}}{\sum_{i=1}^{N_{g}} H_{i}} \\
\bar{\omega}=\frac{\sum_{i=1}^{N_{g}} \omega_{i} H_{i}}{\sum_{i=1}^{N_{g}} H_{i}} \\
\frac{d \bar{\omega}_{\Delta}}{d t}=-\frac{P_{\Delta}}{2 \sum_{i=1}^{N_{g}} H_{i}}
\end{array}
$$

where $\frac{d \bar{\omega}_{\Delta}}{d t}$ is the mean retardation of all machines in a power system after a disturbance, $P_{\Delta}$ is the power imbalance due to the disturbance, $H_{i}, \delta_{i}$ and $\omega_{i}$ are the inertia constant, angle and angular velocity of machine $i$ respectively and $N_{g}$ is the total number of machines in the power system. It is emphasised in [8] that the system as a whole will retard at a rate given by the inertial centre. However, the individual machines will retard at different rates governed by each machines individual swing equation, and only after a transient period will all the machines retard at the same rate. This inertial centre is often used to determine the system RoCoF of a power system after a disturbance. However, it only considers the system rate of change of speed and does not take account of the initial rate of change of speed of the individual machines immediately after a disturbance.

It is important to note that equation 3 calculates the system angular velocity using a weighted sum of the angular velocities of the individual generators. The weight applied to the angular velocity of each generator is equal to the proportion of the total power system inertia at that generator, hence, generators with more inertia have more influence on the system frequency and RoCoF. 


\section{Methodology}

\section{A. General Overview}

To investigate the local nature of RoCoF, a model of the New England Power System, also known as the IEEE 39 bus power system, was implemented and simulated using the software package Power Systems Simulation for Engineering (PSS/E). The IEEE 39 bus power system consists of 10 generators, 19 loads and 39 buses.

Five scenarios with different distributions of inertia were investigated. For scenarios 1, 4 and 5, the simulation was run 10 times, each time a different machine was tripped from the system. In scenarios 2 and 3 only machine 5 was tripped. Machine rotor speeds and bus frequencies were recorded over the simulated duration of 5 seconds. Machine governors were disabled so that they would not influence the results as the objective was to observe the natural behaviour of the system.

PSS/E does not have the facility to calculate RoCoF and so the bus frequency and machine speed data were exported to Matlab for analysis. The local RoCoF at each generator bus was calculated and compared to the electrical distance, measured in terms of the synchronising torque, to the tripped machine. The system RoCoF for each simulation was also calculated.

\section{B. Scenarios}

Table I gives the details of the distributions of inertia for each scenario. Scenarios 1, 2 and 3 were used to investigate how the RoCoF in a power system varies with location, while scenarios 1,4 and 5 were used to investigate the effect that the distribution of inertia has on the RoCoF. Scenario 1 has an equal distribution of inertia across all machines. In scenarios 2 and 3, machine 5 was tripped while the electrically closest machine to 5, number 4 , had half and then double the inertia of the remaining machines. In scenarios 4 and 5, 68\% and $90 \%$ of the total power system inertia was concentrated at bus 39 (machine 10).

TABLE I

DISTRIBUTION OF INERTIA FOR SCENARIOS 1 TO 5. INERTIA CONSTANTS ARE EXPRESSED ON A SYSTEM BASE OF 100 MVA

\begin{tabular}{|c|c|c|c|c|c|}
\hline $\begin{array}{c}\text { Inertia } \\
\text { No. }\end{array}$ & $\begin{array}{c}\text { Scenario } \\
1\end{array}$ & $\begin{array}{c}\text { Scenario } \\
2\end{array}$ & $\begin{array}{c}\text { Scenario } \\
3\end{array}$ & $\begin{array}{c}\text { Scenario } \\
4\end{array}$ & $\begin{array}{c}\text { Scenario } \\
5\end{array}$ \\
\hline H1 & 87.5 & 92.6 & 78.7 & 11.6 & 9.7 \\
\hline H2 & 87.5 & 92.6 & 78.7 & 25.3 & 9.7 \\
\hline H3 & 87.5 & 92.6 & 78.7 & 30.2 & 9.7 \\
\hline H4 & 87.5 & 46.3 & 157.5 & 33.6 & 9.7 \\
\hline H5 & 87.5 & 87.5 & 87.5 & 28.1 & 9.7 \\
\hline H6 & 87.5 & 92.6 & 78.7 & 37.8 & 9.7 \\
\hline H7 & 87.5 & 92.6 & 78.7 & 27.1 & 9.7 \\
\hline H8 & 87.5 & 92.6 & 78.7 & 23.6 & 9.7 \\
\hline H9 & 87.5 & 92.6 & 78.7 & 58.1 & 9.7 \\
\hline H10 & 87.5 & 92.6 & 78.7 & 599.5 & 787.3 \\
\hline Total & 874.8 & 874.8 & 874.8 & 874.8 & 874.8 \\
\hline
\end{tabular}

\section{Local RoCoF and System RoCoF}

Immediately after a power system disturbance, a deviation will exist in the frequency and RoCoF between all buses until the synchronous generators return to a coherent state. This will produce local variations in frequency and RoCoF throughout the system. The term local is used to describe the frequency and RoCoF observed at an actual bus. The term system is used to describe the frequency and RoCoF of the power systems centre of inertia.

To calculate the local RoCoF at the generator buses the following equation was implemented in Matlab:

$$
\frac{d f}{d t}[n]=\frac{f[n]-f[n-N]}{N T}
$$

Where $\frac{d f}{d t}[n]$ is the RoCoF at sample $n, f[n]$ is the frequency at sample $n, N$ is the number of samples in the moving average window and $T$ is the simulation time step size.

The simulations showed that at some buses the highest RoCoF was not always immediately after the disturbance. Sometimes the highest RoCoF at a particular bus occurred some time later. For this reason, all simulated RoCoF results are the highest local or system RoCoF observed and not necessarily the RoCoF immediately after the disturbance.

To calculate the system RoCoF, the system frequency was calculated using equation 3 and then the system RoCoF was calculated by applying equation 5 to the system frequency.

\section{RoCoF and RoCoRS}

When simulating disturbances on electrical power systems using PSS/E, the dynamic frequency of each bus may not be accurate at the instant and immediately after the disturbance. This is a problem especially when trying to calculate the instantaneous RoCoF immediately after a disturbance.

The method of dynamic frequency calculation implemented by PSS/E is based on the derivative of the bus voltage angles and this can result in an unrealistic frequency for certain types of disturbances [11]. This is because immediately after a disturbance, the bus voltage phase angle may change instantly, resulting in a very large derivative. This is a common issue with power system simulation software [12].

To avoid the possibility of inaccurate results, and since the frequency of a generator bus should be the same as the rotational frequency of the generator rotor, we decided to use the rate of change of rotor speed (RoCoRS) as a more reliable indication of the local frequency and RoCoF immediately after a disturbance. However, the disadvantage of this assumption is that the results are limited to the generator buses only and the local dynamic frequency and $\mathrm{RoCoF}$ of non-generator buses has not been considered.

\section{E. Electrical Distance}

To investigate how the magnitude of the local RoCoRS varies with electrical distance from the disturbance, the electrical distance from each generator to all other generators, was calculated and expressed in terms of the synchronising torque 


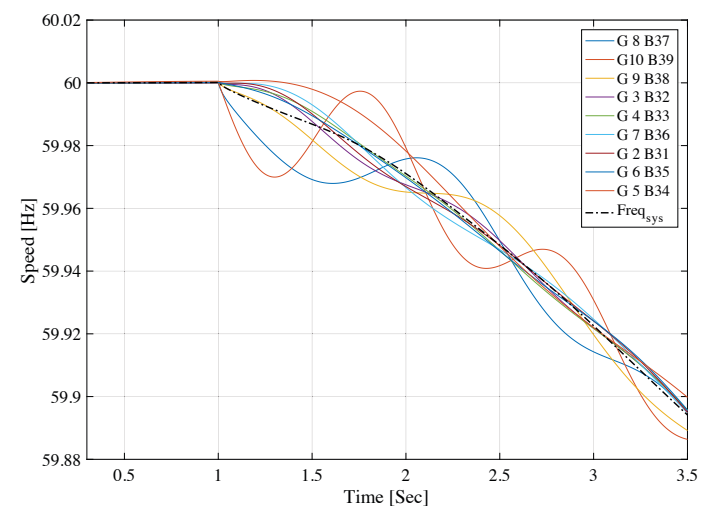

Fig. 1. Remaining machines' rotor speeds after generator 1 trips.

between each machine. A similar method is demonstrated in [8].

1) All loads were converted to their equivalent admittances.

2) All generator internal emfs were calculated using the load flow data and the generator transient reactance values.

3) The admittance matrix for the power system was modified so that the internal nodes of the generators were part of the admittance matrix.

4) All nodes in the admittance matrix, except internal generator nodes, were eliminated using the Kron matrix reduction technique.

5) The synchronising torque between each machine was calculated.

6) The synchronising torque between each machine and the tripped machine was expressed as a percentage of the sum of synchronising torque from all machines to the tripped machine.

\section{Results AND Discussion}

Figures 1 and 2 show the results of one of the simulations from scenario 1. Machine number 1 was tripped at a time of 1 second. Figure 1 shows the change in rotor speeds for the remaining 9 machines following the disturbance. It can be seen that all machines begin to decelerate and oscillate, some at a different rates than others. It is obvious that machines G8 and G10 have been impacted the most and will have the largest RoCoRS. Figure 2 shows the RoCoRS for all the remaining machines.

Figure 3 shows the most severe RoCoRS seen at each of the remaining machines after tripping machine 1 . The figure also shows the percentage of the total synchronising torque between the remaining machines and the tripped machine. It can be clearly seen that there is a strong correlation between the magnitude of the RoCoRS of each machine and the percentage of the total synchronising torque between each machine and the tripped machine. The greater the percentage of total synchronising torque between a remaining machine

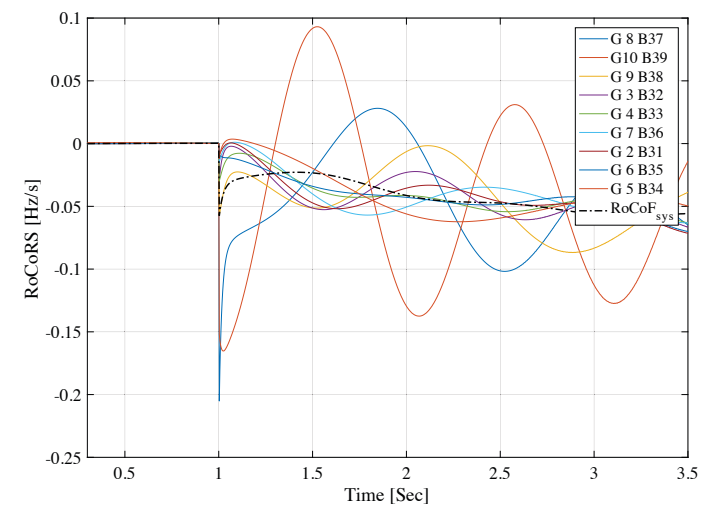

Fig. 2. Remainig machines' RoCoRS after generator 1 trips.

and the tripped machine, the more severe the RoCoRS will be for that machine. However, this is on the basis that all machines have equal inertia. The magnitude of the RoCoRS of an individual machine can also be expressed as a percentage of the mean of the RoCoRS across all machines. Figure 3 shows that the maximum RoCoRS was seen at machine 8 , with a magnitude of $212 \%$, expressed as a percentage of the mean of the RoCoRS across all machines.

Figure 4 shows the maximum RoCoRS seen in each of the 10 simulations from scenario 1 . Each RoCoRS is expressed as a percentage of the mean of all the RoCoRS for that particular simulation. Figure 4 also shows the percentage of the total synchronising torque between the machines with the highest RoCoRSs and the tripped machine for each of the simulations. There is a strong correlation between the magnitude of the relative RoCoRS of each machine and the percentage of the total synchronising torque between each machine and the tripped machine. It can be observed that machines with a high synchronising torque to the tripped machine, relative to the remaining machines, also have a relatively high RoCoRS.

Figure 4 shows that when machine 5 was tripped, generator 4 experienced the second highest RoCoRS from all the simulations at $345 \%$ of the mean of all the RoCoRS for that particular simulation. This machine has a very high percentage of the total synchronising torque to the tripped machine, approximately $65 \%$. To explore this further, scenarios 2 and 3 were simulated. Machine 5 was tripped in both scenarios. However, the distribution of the inertia of the remaining machines was different. For the second and third scenarios, the inertia of the remaining machines was arranged so that machine 4 , i.e. the machine with the relatively highest synchronising torque to the tripped machine, had half and then double the inertia of the remaining machines. All other remaining machines had equal inertia.

Table II shows the results of the three scenarios after machine 5 was tripped. The results of scenario 1 show that machine 4 has the greatest share of the synchronising torque and is impacted the most as its RoCoRS is $-0.88 \mathrm{~Hz} / \mathrm{s}$. This 
is considerably greater than the other remaining machines. In scenarios 2 and 3, machine 4 has 50\% less and 100\% more inertia compared to the remaining machines respectively, and as a consequence the RoCoRS seen in both scenarios is $1.67 \mathrm{~Hz} / \mathrm{s}$ and $-0.49 \mathrm{~Hz} / \mathrm{s}$ respectively. The RoCoRS seen at the other remaining machines in both scenarios did not change significantly. This demonstrates that the RoCoRS for a particular machine is dependent on the proportion of its synchronising torque to the tripped machine and also on its proportion of inertia.

Figure 5 shows the distribution of the RoCoRS results for scenarios 1, 4 and 5. For clarity only the results of the simulations where machines 1, 3 and 9 were tripped are shown. This figure shows how the distribution of the RoCoRS results change as the distribution of the inertia changes. The box and whisker diagrams show the distribution of RoCoRS results, the mean of the RoCoRSs (diamond), the minimum and maximum RoCoRS (ends of the whiskers), median (red horizontal line) and system RoCoF (asterisk) for nine simulations, three from each scenario. The results show that when the inertia is equally distributed (scenario 1), the mean of the RoCoRS and the system RoCoF are almost exactly the same. As the inertia becomes more concentrated to a part of the power system (scenarios 4 \& 5), the system RoCoF remains unaffected. However the magnitude of the mean of the RoCoRS becomes larger. It can also be seen from the results that as the inertia becomes more concentrated to parts of the power system, the magnitude of the most severe RoCoRS also becomes larger.

As inertia distribution is changed for different scenarios, generators with more inertia have a lower RoCoRS but have more influence on the system RoCoF, generators with less inertia have a larger RoCoRS but less influence on the system RoCoF, so the net effect for different distributions of inertia is that the dynamic system frequency and rocof is always the same. However, the distribution of inertia does have an effect on the mean of the RoCoRSs as this is an un-weighted average. This could be an issue because protective equipment measures local RoCoF and not system RoCoF.

Figure 6 shows the results of the same simulations as figure 5 , except the RoCoRS is measured over a 0.5 second rolling average window. It can be seen that the magnitude of the measured RoCoRS is significantly lower and closer to the system RoCoRS. However, for some of the simulations there is still a significant difference between the system $\mathrm{RoCoF}$ and the most severe RoCoRS. When machine 9 was tripped in scenario 5, the system RoCoF was recorded as approximately $-0.21 \mathrm{~Hz} / \mathrm{s}$ but the most severe RoCoRS was recorded as approximately $-0.56 \mathrm{~Hz} / \mathrm{s}$, almost 2.7 times larger than the system RoCoF. The results shown in figure 6 also suggest that distribution of inertia has less of an effect on the measured RoCoRS when measured using a 0.5 second rolling window.

\section{CONCLUSION}

The results of the simulations have shown that when a large disturbance occurs on a power system, such as the loss of a generator, all remaining generators will be impacted

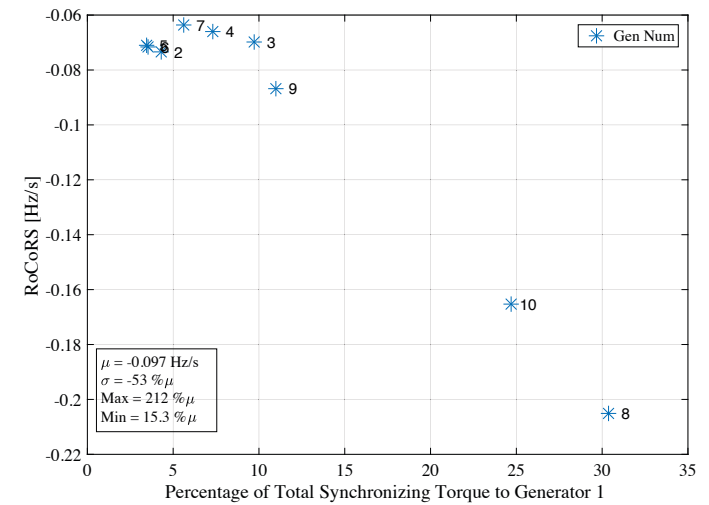

Fig. 3. Generators' RoCoRS after generator 1 trips, scenario 1.

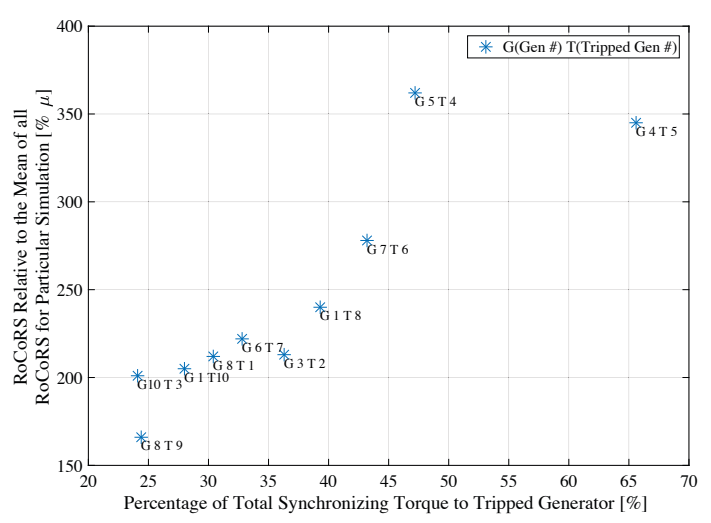

Fig. 4. Most severe RoCoRS from each simulation.

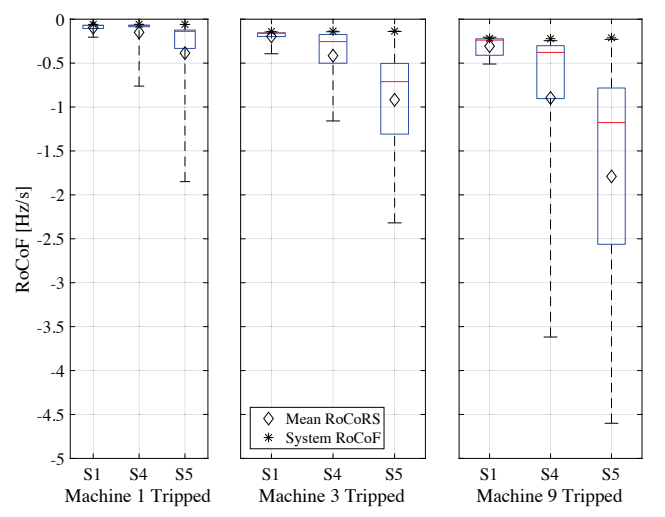

Fig. 5. Distribution of RoCoRS results for scenarios $1,4 \& 5$.

to a greater or lesser extent. The generator closest to the disturbance, measured in terms of the synchronising torque, will experience the greatest impact and if this machine has 


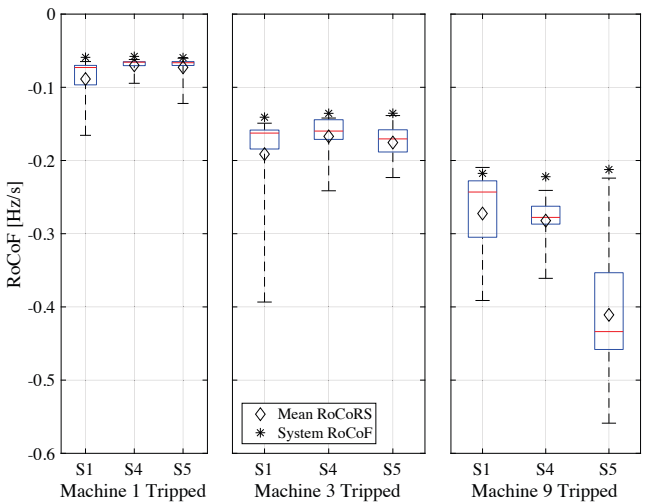

Fig. 6. Distribution of RoCoRS results for scenarios $1,4 \& 5$ measured over a 0.5 second rolling window.

TABLE II

ROCORS FOR ALL GENERATORS AFTER LOSS OF GENERATOR NO. 5

\begin{tabular}{|c|c|c|c|c|}
\hline \multirow{2}{*}{$\begin{array}{l}\text { Machine } \\
\text { No. }\end{array}$} & \multirow{2}{*}{$\begin{array}{l}\text { Share of Syn- } \\
\text { chronising } \\
\text { Torque [\%] }\end{array}$} & \multicolumn{3}{|c|}{ RoCoRS [Hz/s] } \\
\cline { 3 - 5 } & 65.6 & -0.88 & -1.67 & -0.49 \\
\hline 4 & 7.9 & -0.24 & -0.17 & -0.19 \\
\hline 7 & 5.1 & -0.16 & -0.16 & -0.16 \\
\hline 3 & 5 & -0.20 & -0.19 & -0.19 \\
\hline 6 & 4.4 & -0.16 & -0.17 & -0.18 \\
\hline 1 & 3.5 & -0.17 & -0.24 & -0.17 \\
\hline 9 & 3.5 & -0.15 & -0.17 & -0.18 \\
\hline 10 & 3 & -0.16 & -0.17 & -0.17 \\
\hline 8 & 1.9 & -0.17 & -0.18 & -0.20 \\
\hline 2 & & & & \\
\hline
\end{tabular}

inertia equal to or less than the other remaining machines, it will experience a RoCoRS possibly many times greater than the system RoCoF. This highlights a possible dangerous scenario when operating electrical power systems. If a single machine, with low inertia relative to the other machines, has a high percentage of the total synchronising torque to a machine that is suddenly tripped, it could experience a very high RoCoRS, potentially causing its protective devices to operate and disconnecting it from the network.

It was also observed that the distribution of the inertia does not have an affect on the magnitude of the system RoCoF following a disturbance. However it does have a significant effect on the mean of the RoCoRSs. This in turn would have an effect on the local frequency and RoCoF during the transient conditions following a disturbance. Anti-islanding RoCoF relays and generator protection equipment measure and operate based on local frequency and $\mathrm{RoCoF}$ and not the system frequency and system RoCoF. The implication of these results are that as the power system inertia becomes concentrated to a part of the power system, following a disturbance, some of the local RoCoFs may be far greater than the system RoCoF and this increases the probability of the operation of protection equipment which could further compound the initial disturbance.

Measuring RoCoF using a moving average filter over a 0.5 second rolling window can significantly reduce the measured $\mathrm{RoCoF}$ and this would decrease the probability of nuisance tripping of protective equipment. Also, when measured using a rolling window, the distribution of inertia has less effect on the measured local RoCoF. However, the results of the simulations did demonstrate that even when RoCoF is measured using a rolling window, the most severe local RoCoF can still be significantly larger than the system RoCoF.

Electrical power systems must be designed and operated in such a way as to ensure that following a large disturbance, not only the system RoCoF, but also the local RoCoFs are below the limits of the system. The results of the research suggest that maintaining a minimum amount of inertia connected to a power system will ensure that following a disturbance the system RoCoF may stay within limits. However, local RoCoFs may be significantly larger than expected and breach limits.

\section{REFERENCES}

[1] G. Lalor, J. Ritchie, S. Rourke, D. Flynn, and M. J. O'Malley, "Dynamic frequency control with increasing wind generation," in 2004 IEEE Power Engineering Society General Meeting. IEEE, pp. 1715-1720.

[2] N. Jenkins, R. Allan, P. Crossley, D. Kirschen, and G. Strbac, Embedded Generation. IET, Jan. 2000.

[3] EirGrid \& SONI. (2012, Sep.) RoCoF Modification Proposal [Online]. Available: http://www.soni.ltd.uk/media/documents/Archive/ RoCoF\%20Modification\%20Proposal\%20TSOs\%20Opinion.pdf [Accessed: 29/06/2016]

[4] EirGrid \& SONI. (2010, Jun.) All Ireland TSO Facilitation of Renewables Studies. [Online]. Available: http://www.eirgrid.com/ renewables/facilitationofrenewables/ [Accessed: 14/10/2015]

[5] EirGrid \& SONI. (2017, Apr.) Annual Renewable Energy Constraint and Curtailment Report 2016. [Online]. Available: http://www.eirgridgroup.com/site-files/library/EirGrid/AnnualRenewable-Constraint-and-Curtailment-Report-2016-v1.0.pdf [Accessed: 27/05/2017]

[6] K. Creighton, M. McClure, R. Skillen, J. O' Higgin, T. McCartan, and A. Rogers. Increased Wind Generation in Ireland and Northern Ireland and the Impact on Rate of Change of Frequency. [Online]. Available: http://www.eirgridprojects.com/sitefiles/library/EirGrid/Increased $\% 20$ Wind $\% 20$ Generation $\% 20 \mathrm{in} \%$ 20Ireland $\% 20$ and $\% 20$ Northern $\% 20$ Ireland $\% 20$ and $\% 20$ the $\% 20$ Impact $\%$ 20on $\% 20$ Rate $\% 20$ of $\% 20$ Change $\% 20$ of $\% 20$ Frequency.pdf [Accessed: $14 / 11 / 2016]$

[7] D. Wu, M. Javadi, and J. N. Jiang, "A preliminary study of impact of reduced system inertia in a low-carbon power system," Journal of Modern Power Systems and Clean ..., vol. 3, no. 1, pp. 82-92, 2015.

[8] P. M. Anderson and A. A. Fouad, Power System Control and Stability, 2nd ed. IEEE Press: John Wiley \& Sons, 2003.

[9] O. Dudurych and M. Conlon, "Impact of Reduced System Inertia as a Result of Higher Penetration Levels of Wind Generation," in Power Engineering Conference (UPEC), 2014 49th International Universities. IEEE, 2014, pp. 1-6.

[10] EirGrid. (2015, Jul.) EirGrid Grid Code. [Online]. Available: http://www. eirgridgroup.com/site-files/library/EirGrid/GridCodeVersion6.pdf [Accessed: 05/03/2017]

[11] G. Radman and M. A Tabrizi, "Calculation of Power System Dynamic Frequency during Simulation Phase," International Journal of Emerging Electric Power Systems, vol. 13, no. 5, 2012.

[12] F. Milano and A. Ortega, "Frequency Divider," IEEE Transactions on Power Systems, vol. 32, pp. 1493-1501. 